\title{
Nationwide survey on predictive genetic testing for late-onset, incurable neurological diseases in Japan
}

\author{
Kunihiro Yoshida · Takahito Wada • Akihiro Sakurai • \\ Keiko Wakui · Shu-ichi Ikeda · Yoshimitsu Fukushima
}

Received: 15 February 2007/ Accepted: 12 June 2007/Published online: 5 July 2007

(C) The Japan Society of Human Genetics and Springer 2007

\begin{abstract}
A nationwide survey was conducted for predictive genetic testing for late-onset, incurable neurological diseases. A questionnaire was sent to 125 university hospitals and national hospitals, and was returned by $69 \%$ of them. Of the 86 responding hospitals, 63 had genetic counseling clinics and answered the questions concerning predictive testing. Of these, 46 had experienced clients with an interest in or a request for predictive testing during the period from April 2004 to March 2006. A total of 322 clients were accumulated, the majority of which were interested in myotonic dystrophy $(n=150)$, followed by spinocerebellar ataxia $(n=86)$, spinal and bulbar muscular atrophy $(n=40)$ and Huntington's disease $(n=31)$. Most such clients were counseled by medical doctors, who had the "Japanese Board of Medical Genetics, Clinical Geneticist" certification, but others, including neurologists, nurses, clinical psychologists or genetic counselors also contributed, albeit to a lesser extent, to genetic counseling in Japan. Many respondents felt that a multidisciplinary approach by a counseling team consisting of a clinical geneticist, a neurologist, a genetic nurse, a clinical
\end{abstract}

K. Yoshida $(\bowtie) \cdot$ S. Ikeda

Depertment of Neurology and Rheumatology,

Shinshu University Hospital, 3-1-1 Asahi,

Matsumoto 390-8621, Japan

e-mail: kyoshida@hsp.md.shinshu-u.ac.jp

K. Yoshida · T. Wada · A. Sakurai · K. Wakui · Y. Fukushima Division of Clinical and Molecular Genetics,

Shinshu University Hospital, Matsumoto, Japan

T. Wada · A. Sakurai - K. Wakui · Y. Fukushima

Department of Preventive Medicine,

Shinshu University School of Medicine,

Matsumoto, Japan psychologist and a genetic counselor had not yet been established. There will be a great need for educated and trained non-medical doctor staff not only to improve the quality of genetic counseling and psychological support for such clients, but also to conduct the psychosocial research on Japanese clients requesting predictive genetic testing.

Keywords Genetic counseling - Predictive genetic testing $\cdot$ Neurological disease $\cdot$ Clinical geneticist

\section{Introduction}

In Japan, genetic counseling has not yet taken root as a common clinical practice, but genetic testing and genetic research for human diseases have become increasingly popular. Along with this trend, the establishment of a genetic counseling system is strongly needed to successfully conduct clinical practice and research for genetic diseases. In recent years, specific genetic counseling departments have been set up nationwide. Now more than 60 hospitals have opened genetic counseling clinics in Japan. The National Liaison Meeting for Clinical Sections of Medical Genetics has been held annually since 2003, where the persons responsible for genetic counseling departments have gathered from throughout the country and held discussions on several issues concerning the genetic counseling system in Japan.

The most controversial topic in clinical genetics is how we should handle the need for predictive and prenatal genetic testing, especially, predictive testing for late-onset, incurable neurological diseases such as Huntington's disease (HD) and spinocerebellar ataxia (SCA). It raises various ethical, legal and psychosocial implications (Brandt 
1994; Evers-Kiebooms and Decruyenaere 1998; Chapman 2002; Taylor 2004); thus, pre-test genetic counseling and post-test follow-up should be carefully conducted for clients.

Predictive testing for late-onset, incurable neurological diseases has become popular in the United States, Canada, Australia and various European countries, and integrated guidelines and protocols for predictive testing have been established in these countries (Craufurd et al. 1992; Quaid 1992; International Huntington Association (IHA) and World Federation of Neurology (WFN) 1994; Benjamin et al. 1994; Decruyenaere et al. 1995; Mandich et al. 1998; Robins Wahlin et al. 2000). However, little is known about the actual circumstances surrounding this issue in Japan, and only a few studies on psychological aspects of clients seeking predictive testing have been published (Abe and Itoyama 1997; Muto 1998; Yoshida et al. 2002).

In this survey, we investigated the current situations and attitudes of genetic counseling departments in Japan concerning predictive testing for late-onset, incurable neurological diseases.

\section{Procedure}

We sent the questionnaire to 125 institutions (university hospitals and national hospitals) in Japan, most of which were highly advanced and specialized medical center hospitals under the supervision of the Ministry of Health, Labor and Welfare, to investigate the actual circumstances concerning predictive testing for late-onset, incurable neurological diseases. The diseases we indicated in the questionnaire included $\mathrm{HD}, \mathrm{SCA}$, amyotrophic lateral sclerosis (ALS), Alzheimer's disease (AD), spinal and bulbar muscular atrophy (SBMA), myotonic dystrophy (DM1) and prion disease. Familial amyloid polyneuropathy (FAP) was also included, although it may no longer be incurable due to liver transplantation. Before submitting the questionnaire, we asked whether or not the hospital had a specific genetic counseling department. If not, the responding persons were instructed not to answer the questions about predictive testing. The questionnaire contained five questions as shown in Table 1. The chief person responsible for the genetic counseling department in each hospital was asked to fill out the questionnaire. In this paper, a clinical geneticist indicates a medical doctor who has the "Japanese Board of Medical Genetics, Clinical Geneticist" certification, and a genetic counselor indicates a non-medical doctor who has the "Japanese Board of Medical Genetics, Certified Genetic Counselor" certification. The certificates are given by the Japan Society of Human Genetics and the Japanese Society for Genetic Counseling.

\section{Results}

The questionnaire was returned by $69 \%(n=86)$ of the hospitals surveyed. Of the 86 responding hospitals, 63 (73\%) had clinics that specialized in genetic counseling and answered the questions concerning predictive testing as shown in Table 1. The distribution of these 63 hospitals is shown in Fig. 1. The remaining 23 hospitals did not have genetic counseling clinics. Of the 63 hospitals with clinics, $46(73 \%)$ had clients with an interest in or a request for predictive testing for late-onset, incurable neurological diseases during the period from April 2004 to March 2006. Of these 46 hospitals, $30(65 \%)$ were attached to national universities or national centers, $13(28 \%)$ were private universities, and $3(7 \%)$ were public universities. The remaining 17 hospitals had no requests or inquiries for predictive genetic testing during this period.

In total, 322 clients from 243 families (excluding FAP) were reported from the 46 responding hospitals (Table 1 Q2). National and public hospitals (total 33) had 194 clients, and private hospitals (total 13) had 128 clients. The maximum number of clients was 80 in a hospital located in the Kanto area. Eleven hospitals had more than 10 clients, and 27 had less than 5 clients during this period. The greatest interest was shown in DM1 (150 clients, 47\%), followed by SCA (86 clients, 27\%), SBMA (40 clients, $12 \%$ ) and HD (31 clients, 10\%). These clients were distributed nationwide, and more than ten of the hospitals had clients interested in each of the following four diseases (DM1: 31 hospitals; SCA: 30; SBMA: 10; HD: 14). Those interested in prion disease (13 clients, 4\%) were much fewer and were found in only three hospitals; a single hospital reported 10 of the 13 clients for prion disease. The interest in ALS was very small ( 2 clients, $<1 \%$ ) and was found in only two hospitals. There were no clients reported to be interested in $\mathrm{AD}$ during this period. There was a significant number of clients (more than 13 clients from 11 families) interested in FAP, but the exact number was not obtained because the answer "a lot" was returned from a single hospital. In contrast to the clients for DM1 or SCA, those for FAP were exclusively concentrated in two hospitals that are close to the former endemic areas for this disease and that have actively conducted liver transplantations for the patients.

The professions of the participants in genetic counseling sessions are summarized in Table 1 (Q3). Clinical geneticists participated in $75-100 \%$ of the total counseling sessions in 35 of the hospitals $(56 \%)$. The contributions of neurologists and nurses were much smaller; they participated in 75-100\% of counseling sessions in just 14 and 13 hospitals, respectively. The participation of the other professions (psychiatrists, clinical psychologists, genetic counselors, etc.) was not common. Seventeen 
Table 1 Questionnaire and results
Have you had clients who visited your hospital with an interest in or a request for Q1 predictive genetic testing for late-onset, incurable neurological diseases during the period from April 2004 to March 2006? (total responding = 63)

\begin{tabular}{|l|l|}
\hline Yes; (go to Q2-5) & 46 \\
\hline No; (go to Q4-5) & 17 \\
\hline
\end{tabular}

What kinds of diseases were your clients interested in? Please indicate the numbers of

families (clients) for each disease. (total responding $=46$ )

\begin{tabular}{|l|l|}
\hline Myotonic dystrophy & $110(150)$ \\
\hline Spinocerebellar ataxia & $68(86)$ \\
\hline Spinal and bulbar muscular atrophy & $32(40)$ \\
\hline Huntington's disease & $26(31)$ \\
\hline Prion disease & $5(13)$ \\
\hline Amyotrophic lateral sclerosis & $2(2)$ \\
\hline
\end{tabular}

How much did the following professions contribute to the counseling sessions of such

clients? Please select the most appropriate percentage among the following: a: $0 \%$, b:

Q3 $0-25 \%$, c: $\mathbf{2 5 - 5 0} \%$, d: $\mathbf{5 0 - 7 5 \%}$, and e: $\mathbf{7 5 - 1 0 0 \% ~ ( ' 0 \% ' ~ i n d i c a t e s ~ n o ~ p a r t i c i p a t i o n ~ a n d ~}$ ' $100 \%$ ' indicates full-time participation in the counseling sessions.)

\begin{tabular}{|l|c|c|c|c|c|}
\hline & $\mathbf{0 \%}$ & $\mathbf{0 - 2 5 \%}$ & $\mathbf{2 5 - 5 0 \%}$ & $\mathbf{5 0 - 7 5 \%}$ & $\mathbf{7 5 - 1 0 0 \%}$ \\
\hline Clinical geneticist (46) & 2 & 1 & 4 & 4 & 35 \\
\hline Neurologist (41) & 10 & 7 & 7 & 3 & 14 \\
\hline Psychiatrist (33) & 26 & 3 & 3 & 0 & 1 \\
\hline Nurse (40) & 13 & 5 & 6 & 3 & 13 \\
\hline Clinical psychologist (37) & 17 & 6 & 6 & 0 & 8 \\
\hline Genetic counselor (28) & 26 & 0 & 0 & 1 & 1 \\
\hline
\end{tabular}

If such clients visit your department in the future, how will you handle them? Please

select the most appropriate answer from the following: (total responding $=63$ )

\begin{tabular}{|l|l} 
You will provide genetic counseling in your own department & 36
\end{tabular}

You will refer the client to another hospital without providing genetic

counseling yourself

You will make your decision ( 1 or 2 shown above) depending on the

situation and/or disease of interest of the client

You will reject the client

Not answered

2

What do you think are the most important requirements for maintaining genetic counseling for such clients? Please select the three most important requirements from the following and rank them in the order of their importance, (1st) being most important. (total responding $=63$ )

\begin{tabular}{|l|l|l|l|}
\hline & \multicolumn{1}{|c|}{$\mathbf{1 s t}$} & \multicolumn{1}{|c|}{ 2nd } & \multicolumn{1}{|c|}{ 3rd } \\
\hline Genetic counseling staff & 33 & 12 & 7 \\
\hline Time and space for clinical practice of genetic counseling & 5 & 21 & 6 \\
\hline Administrative support for genetic counseling & 10 & 8 & 8 \\
\hline Genetic testing system & 5 & 9 & 17 \\
\hline Cooperation with the other hospitals & 1 & 7 & 12 \\
\hline Understanding and recognition of clinical genetics by the general & 8 & 3 & 7 \\
\hline Others & 1 & 3 & 6 \\
\hline
\end{tabular}
indicate those of responding hospitals
Concerning the attitudes toward such clients in the future (Table 1 Q4), 97\% of the responding hospitals $(n=61)$ showed positive attitudes toward these clients. Thirty-six hospitals $(57 \%)$ indicated that they would conduct genetic counseling in their own hospitals. Two (3\%) would refer the (19\%). 


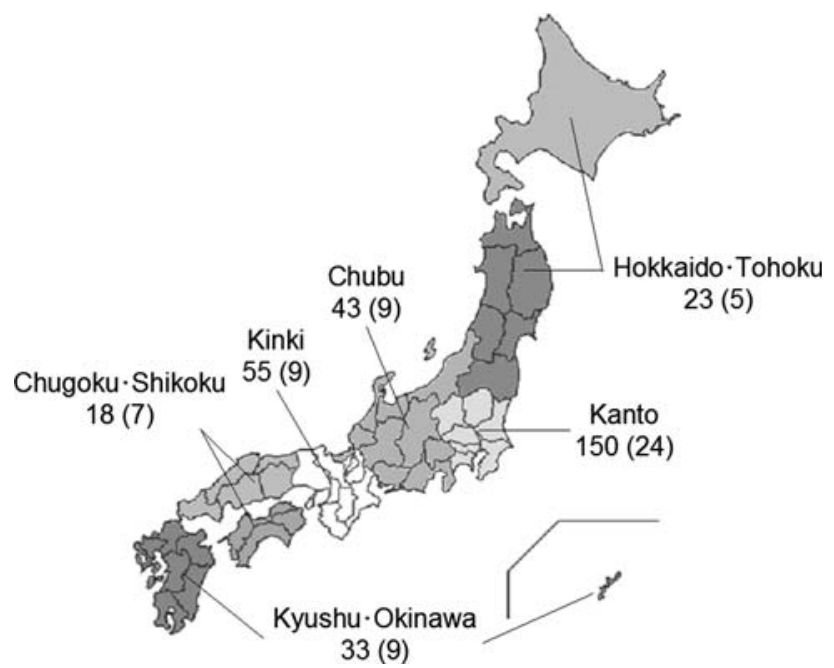

Fig. 1 The distribution and numbers of clients and responding hospitals. The numbers of clients shown here indicate the sum of those with an interest in or request for predictive testing for DM1, SCA, SBMA, HD, prion disease and ALS, but not for FAP. The numbers of responding hospitals in each district are indicated in the parentheses

clients to other hospitals without counseling them themselves, and 23 (37\%) would make these decisions based on the individual clients.

We inquired about the needs of persons involved in the genetic counseling of such clients (Table 1 Q5). The most important requirement was an improvement in the counseling staff, followed by improvements in the environment of clinical practice for genetic counseling and the establishment of genetic testing systems. Administrative or financial support for genetic counseling and cooperation with other hospitals in genetic counseling were also strongly needed.

\section{Discussion}

The number of genetic counseling departments in hospitals has been increasing in Japan in recent years. This was the first nationwide survey of such departments conducted concerning predictive testing for late-onset, incurable neurological diseases. Inherent in such procedures are several ethical and social issues (Brandt 1994; Evers-Kiebooms and Decruyenaere 1998; Chapman 2002; Taylor 2004), and little information on the present conditions surrounding this issue has been accumulated in Japan (Abe and Itoyama 1997; Muto 1998; Yoshida et al. 2002).

It is obvious that a significant number of the clients with an interest in or a request for predictive testing for lateonset, incurable neurological diseases visited hospitals over last 2 years. The clients were not concentrated in specific districts of Japan, but were distributed nationwide. We should keep in mind that the numbers of clients reported in this paper may represent an underestimate, because not all hospitals and clinics were surveyed. Even in the hospitals we did survey, some respondents indicated that neurology departments in their hospitals have such clients independently from genetic counseling departments. Considering that several delicate and complicated issues are involved with predictive testing for the diseases under study, however, it is likely that the clients have concentrated in the hospitals that have specialized genetic counseling clinics. Thus, we presume that the numbers of clients in this paper roughly reflect the current situations on this matter.

There was a marked difference in the number of clients among hospitals we surveyed. The number of clients may be influenced by several factors. They include the locations of hospitals, the attitudes of counseling staff, the activities of genetic counseling departments and cooperation and specialties of neurologists involved. Academic interest of the neurology department in the university may also be closely associated with the numbers and kinds of diseases of the clients. A representative example for this is seen for FAP, as shown in Results. Of 11 hospitals that had more than 10 clients during the period of interest, nurses and/or clinical psychologists participated $75-100 \%$ of the time in genetic counseling sessions in 7 hospitals, whereas, neither of them participated at all in 2 hospitals. As far as predictive testing for late-onset, incurable, neurological diseases, therefore, the participation of non-medical doctor (nonMD) staff in the counseling session does not seem to directly parallel the number of clients. But we can say that requests of clients for predictive testing might be a driving force for having nurses or clinical psychologists involved in genetic counseling sessions. The type of hospitals (national, public or private) had no effect on the number of clients.

It is reasonable that the number of clients interested in DM1 was much greater than those interested in either HD or SCA. The major reason for this is that predictive testing for DM1 often may be linked with reproductive decision making, based on the fact that prenatal genetic testing for DM1 has often been performed in Japan. Many clinical geneticists and neurologists also feel that the matter of predictive testing for DM1 is far different than for HD or SCA because DM1 can be diagnosed by clinical and laboratory findings other than genetic testing, even when a person is not aware of the symptoms. Thus, a significant portion of DM1 clients who were likely to test negative may have already had genetic testing in Japan. This may also be the case for SBMA, because SBMA can be detected by electromyography at the preclinical stage. Thus, careful consideration should be given to whether different predictive testing procedures should be applied to DM1 or SBMA than to HD, SCA or AD. 
During the period of the survey, clinical geneticists were predominantly involved in genetic counseling sessions for the diseases under study, probably regardless of their own medical specialties. By contrast, the contribution of neurologists was far less than that of clinical geneticists in Japan. Some respondents strongly desired an active participation of neurologists in the counseling team. Neurologists who are involved in genetic testing for patients with hereditary neurological diseases should give much more consideration to the possibility of predictive testing of the patients' family members. The involvement of non-MD staff such as nurses and clinical psychologists was not common in Japan. These results indicate that genetic counseling has been still largely dependent on medical doctors with an interest in clinical genetics and a multidisciplinary approach by a genetic counseling team has not yet functioned effectively in Japan. This situation is mainly because it is very difficult for non-MD staff (nurses, clinical psychologists, genetic counselors) to secure a position for genetic counseling in the hospital in Japan. Especially clinical psychologists, if involved in the genetic counseling team, have been compelled to work under limited conditions. From the viewpoint of hospital management, the cost-benefit performance is poor for genetic counseling, and non-MD staff specific for genetic counseling cannot be employed. In this study, some respondents desired a hospital cost system that profited from genetic counseling.

To improve the present conditions, we first need to have genetic counseling widely recognized in the general population. Then we need to know in more detail how satisfied clients are with genetic counseling sessions and to know what benefit they receive from having non-MD staff in the genetic counseling sessions. A counseling team consisting of a clinical geneticist, a neurologist, a genetic nurse and a clinical psychologist for each session is generally advisable. We believe this format is desired for patient or clientcentered genetic counseling. The education and training of non-MD staff will be of great help not only to maintain the quality of genetic counseling for clients, but also to conduct medical research on the psychosocial aspects of genetic counseling in Japan.

Acknowledgments The authors would like to acknowledge the respondents participating in this study. This work was supported by the Study Group for improving community medicine for patients with intractable diseases from the Ministry of Health, Labor and Welfare (KY, SI), and also by grants from the Ministry of Education, Culture, Sports, Science and Technology (YF).

\section{References}

Abe K, Itoyama Y (1997) Psychological consequences of genetic testing for spinocerebellar ataxia in the Japanese. Eur J Neurol 4:593-600

Benjamin CM, Adam S, Wiggins S, Theilmann JL, Copley TT, Bloch M, Squitieri F, McKellin W, Cox S, Brown SA, Kremer HPH, Burgess M, Meshino W, Summers A, Macgregor D, Buchanan J, Greenberg C, Carson N, Ives E, Frecker M, Welch JP, Fuller A, Rosenblatt D, Miller S, Dufrasne S, Roy M, Andermann E, Prevost C, Khalifa M, Girard K, Taylor S, Hunter A, Goldsmith C, Whelan D, Eisenberg D, Soltan H, Kane J, Shokeir MHK, Gibson A, Cardwell S, Bamforth S, Grover S, Suchowersky O, Klimek M, Garber T, Gardner HA, MacLeod P, Hayden MR (1994) Proceed with care: direct predictive testing for Huntington disease. Am J Hum Genet 55:606-617

Brandt J (1994) Ethical considerations in genetic testing: an empirical study of presymptomatic diagnosis of Huntington's disease. In: Fulford KWM, Gillett G, Soskice JM (eds) Medicine and moral reasoning. Cambridge University Press, London, pp 41-59

Chapman E (2002) Ethical dilemmas in testing for late onset conditions: reactions to testing and perceived impact on other family members. J Genet Counsel 11:351-367

Craufurd D, Tyler A, on behalf of the UK Huntington's Prediction Consortium (1992) Predictive testing for Huntington's disease: protocol of the UK Huntington's Prediction Consortium. J Med Genet 29:915-918

Decruyenaere M, Evers-Kiebooms G, Boogaerts A, Cassiman JJ, Cloostermans T, Demyttenaere K, Dom R, Fryns JP, van den Berghe H (1995) Predictive testing for Huntington's disease: risk perception, reasons for testing and psychological profile of test applicants. Genet Counsel 6:1-13

Evers-Kiebooms G, Decruyenaere M (1998) Predictive testing for Huntington's disease: a challenge for persons at risk and for professionals. Patient Educ Couns 35:15-26

International Huntington Association (IHA), World Federation of Neurology (WFN) (1994) Guidelines for the molecular genetics predictive test in Huntington's disease. Neurology 44:1533-1536

Mandich P, Jacopini G, Di Maria E, Sabbadini G, Chimirri F, Bellone E, Novelletto A, Ajmar F, Frontali M (1998) Predictive testing for Huntington's disease: ten year's experience in two Italian centres. Ital J Neurol Sci 19:68-74

Muto K (1998) Attitudes toward pre-symptomatic testing and social services for families with Huntington's disease in Japan. J Health Care Soc 8:67-82 (in Japanese)

Quaid KA (1992) Presymptomatic testing for Huntington disease: recommendations for counseling. J Genet Counsel 1:277-302

Robins Wahlin T-B, Bäckman L, Lundin A, Haegermark A, Winblad B, Anvret M (2000) High suicidal ideation in persons testing for Huntington's disease. Acta Neurol Scand 102:150-161

Taylor SD (2004) Predictive genetic test decisions for Huntington's disease: context, appraisal and new moral imperatives. Soc Sci Med 58:137-149

Yoshida K, Tamai M, Kubota T, Kawame H, Amano N, Ikeda S, Fukushima Y (2002) Analysis of 14 individuals who requested predictive genetic testing for hereditary neuromuscular diseases. Clin Neurol 42:113-117 (in Japanese) 